\title{
Regulation of Human Aortic Endothelial Cell-derived Mesenchymal Growth Factors by Allogeneic Lymphocytes In Vitro \\ A Potential Mechanism for Cardiac Allograft Vasculopathy
}

\author{
Cynthia R. Wagner, Tony E. Morris, Gary D. Shipley, and Jeffrey D. Hosenpud \\ Immunobiology Research Laboratory of the Oregon Cardiac Transplant Program, Departments of Medicine and Cell Biology, \\ Oregon Health Sciences University, Portland, Oregon 97201; and the Immunology Research Laboratory, \\ Portland Veterans Affairs Medical Center, Portland, Oregon 97201
}

\begin{abstract}
Cardiac allograft vasculopathy is thought to be triggered by an alloreactive response to the donor coronary vasculature, resulting in smooth muscle cell proliferation and ultimate occlusion of the donor coronary arteries. To determine whether allogeneic lymphocytes are capable of regulating endothelial-derived smooth muscle cell (SMC) growth factors, human aortic endothelial cells (HAECs) were exposed to allogeneic lymphocytes. The HAEC-lymphocyte co-cultures were assessed for $(a)$ lymphocyte proliferation in response to the allogeneic HAECs; (b) release of soluble factors that stimulate human aortic SMC proliferation; and (c) alteration of HAEC mRNA levels for a panel of known SMC growth factors. Co-culture conditioned medium increased SMC proliferation, compared to medium conditioned by HAECs alone. HAECs exposed to allogeneic lymphocytes increased their expression of mRNA for basic fibroblast growth factor, transforming growth factors $\alpha$ and $\beta$, and platelet derived growth factor $A$ and $B$ chains. These results demonstrate that allogeneic lymphocytes are capable of inducing HAECs to increase mRNA levels for several mesenchymal growth factors and to release bioactive products capable of stimulating SMC cell proliferation in vitro. Additionally, the data support the hypothesis that alloreactive lymphocytes can stimulate allogeneic donor endothelial cells to produce growth factors that may contribute to the intimal thickening seen in cardiac allograft vasculopathy. (J. Clin. Invest. 1993. 92:1269-1277.) Key words: transplantation immunology • vascular smooth muscle • coronary disease $\bullet$ lymphocytes $\bullet$ chronic rejection
\end{abstract}

\section{Introduction}

Cardiac allograft vasculopathy (CAV) ${ }^{1}$ is the major limitation to long-term survival following cardiac transplantation. It is an

Address correspondence to Dr. Jeffrey D. Hosenpud, Oregon Cardiac Transplant Program (L-462), Oregon Health Sciences University, 3181 S.W. Sam Jackson Park Road, Portland, OR 97201. 993.

1. Abbreviations used in this paper: $\mathrm{CAV}$, cardiac allograft vasculopathy; EC, endothelial cell; HAEC, human aortic endothelial cell; HASMC, human aortic smooth muscle cell; HBFG, heparin binding growth factor; MNC, mononuclear cell; poly $(\mathrm{A})^{+}$RNA, polyadenylated RNA; SI, stimulation index; SMC, smooth muscle cell.

J. Clin. Invest.

(C) The American Society for Clinical Investigation, Inc.

0021-9738/93/09/1269/09 \$2.00

Volume 92, September 1993, 1269-1277 aggressive and obliterative form of coronary artery disease involving the entire length of the coronary tree and can develop in the cardiac allograft over a matter of months following cardiac transplantation (1). CAV is a proliferative vasculopathy and not solely an arteriopathy, as evidenced by intimal hyperplasia in not only the coronary arteries but also in the great vessels as well as in the major coronary veins (2). Due to its diffuse nature, CAV is not amenable to traditional therapeutic approaches to coronary artery disease, such as balloon angioplasty or coronary artery bypass grafting. To date, the only therapy that has been successful is retransplantation.

While the pathogenesis of CAV remains unknown, there is substantial evidence to suggest that $\mathrm{CAV}$ is immunologically mediated and probably represents a form of chronic rejection. $\mathrm{CAV}$ is solely restricted to the allograft $(1,3)$. It is more common in patients who produce antibodies to HLA antigens (4). It is associated with cytomegalovirus infection, which may alter alloantigenicity $(5,6)$, and it can be reproduced in rodent allograft models but does not occur in isograft models ( 7 ). In addition, histopathologic studies have demonstrated a low grade inflammatory process in the subendothelial layer of patients with CAV at autopsy (8).

It is clear that peptide growth factors can act to modulate the proliferation rate of mesenchymal cells by acting in either a positive or negative manner (9-11) and that for optimal proliferation, the concerted action of multiple growth factors is generally required (9). PDGF is a ubiquitous mitogen produced by a number of tissues including endothelium and is a potent smooth muscle cell (SMC) mitogen as well as a chemoattractant (12). These properties, the finding of PDGF transcripts in atherosclerotic plaques (13), and the upregulation of PDGF mRNA in association with hypercholesterolemia (14), have resulted in substantial interest in this mitogen in traditional atherosclerosis.

Another family of growth factors that has been shown to be a positive effector of vascular smooth muscle cell growth (11) is the heparin-binding growth factor/fibroblast growth factor (HBGF) family of mitogens. Seven separate genes of the HBGF family have been identified: acidic and basic fibroblast growth factor (aFGF and bFGF), the int-2 locus, $\mathrm{kFGF} / \mathrm{hst}$, FGF-5, FGF-6, and KGF (keratinocyte growth factor). It is important to note that endothelial cells (EC), specifically bovine aortic and microvascular EC, are capable of producing members of the HBGF family of growth factors $(15,16)$. Finally, both the transforming growth factor, type-beta (TGF- $\beta$ ) and type-alpha (TGF- $\alpha$ ) are potent stimulators of SMC proliferation (11).

The histologic features of CAV, which include an intact endothelial cell layer, a single internal elastic lamina, and the absence of vascular necrosis $(1,3)$, suggest that the mechanism of alloimmunologic vascular attack is more likely endothelial 
activation resulting in the augmented expression of smooth muscle cell growth factors, rather than a frank vasculitic process. To date, however, there is no evidence that endothelial cell activation by allogeneic lymphocytes can ultimately result in a proliferative vasculopathy. Therefore, the following studies were undertaken to determine whether the interaction of allogeneic lymphocytes and aortic endothelial cells $(a)$ increases the level of endothelial cell mRNA for a panel of growth factors known to induce smooth muscle cell proliferation, and (b) causes the release of biologically active smooth muscle cell mitogens.

\section{Methods}

Endothelial and smooth muscle cell isolation and culture. Human aortic endothelial cells (HAECs) and human aortic smooth muscle cells (HASMCs) were isolated, cultured, and characterized as previously described (17). Briefly, segments of human ascending aorta were obtained during organ donation after consent was obtained for the donation of any and all tissues. HAECs were isolated by incubating the aortic tissue in a solution of $0.1 \%$ collagenase (CLS/type $1,152 \mathrm{U} / \mathrm{mg}$; Worthington Biochemical Corp., Freehold, NJ) in RPMI 1640 supplemented with $100 \mathrm{U} / \mathrm{ml}$ penicillin, $100 \mu \mathrm{g} / \mathrm{ml}$ streptomycin, $25 \mathrm{mM}$ Hepes, and $24 \mathrm{mM}$ sodium bicarbonate, $\mathrm{pH}$ 7.2 (Gibco, Grand Island, $\mathrm{NY}$ ), at $37^{\circ} \mathrm{C}$ for $1 \mathrm{~h}$. After the incubation, the HAECs were gently swabbed from the luminal surface of the aorta. The purity of the HAEC cultures was determined by their uniform immunofluorescent staining to Factor VIII-related antigen (A082; Dako Corp. Carpinteria, CA) (17-19) and their uptake of fluorescent-labeled acetylated LDL (DiIAc-LDL; Biomedical Technologies, Inc., Stoughton, MA)(17, 20). All studies were performed using "pooled HAECs" generated by combining an equal number of cells from each of seven separate HAEC cultures. The pooled HAECs were from donors representing a wide range of HLA antigens ( see Table I). The HAECs were cultured in endothelial growth medium (EGM-UV; Clonetics Corp., San Diego, CA ) supplemented with an additional 3\% FCS (Tissue Culture Biologicals, Tulare, CA ) and were used at passages 7-9 to insure adequate numbers of endothelial cells for the studies below.

HASMCs were isolated from the aortic tissue (after removing HAECs) by an additional 1-h incubation at $37^{\circ} \mathrm{C}$ in RPMI 1640 containing collagenase $(0.2 \%)$ and elastase $(0.2 \%, \mathrm{E}-0258,67 \mathrm{U} / \mathrm{mg}$; Sigma Chemical Co. St. Louis, MO), and again swabbing the luminal surface. The identity and purity ( $>95 \%$ ) of the HASMCs were confirmed by staining with the smooth muscle $\alpha$-actin-specific mAb HHF35 (a generous gift by Dr. Allen Gown, University of Washington, Seattle, WA) and by a second smooth muscle-specific $\alpha$-actin mAb (no. 1148818; Boehringer Mannheim Biochemicals, Indianapolis, IN) $(17,21,22)$. HASMCs were cultured in RPMI 1640 supplemented with $20 \%$ FCS.

Experimental design. In each experiment, pooled HAECs were incubated separately with two lymphocyte isolates. One set of HAEC controls (i.e., HAECs cultured without lymphocytes) served as the control in each experiment for both lymphocyte preparations. In each of the experiments, all aspects of the study, including lymphocyte proliferation, conditioned medium generation, and HAEC mRNA isolation, were performed together. A total of 5 experiments, using lymphocytes from 10 individuals, were performed for these studies, resulting in 10 individual experimental and 5 control samples.

Lymphocyte isolation. Lymphocytes were isolated from peripheral blood buffy coats as previously described $(18,23)$. The buffy coats were prepared by the Oregon Trail Chapter of the American Red Cross (Portland, OR) from the blood of 10 normal donors. Briefly, mononuclear cells (MNCs) were isolated by density gradient centrifugation over Ficoll-Hypaque (Pharmacia Fine Chemicals, Piscataway, NJ). The MNCs were recovered from the Ficoll-Hypaque gradients and the monocytes were depleted from the MNCs by adherence to tissue culture flasks. The nonadherent cells were recovered and the monocytes were further depleted by passage of the cells over Sephadex G-10 columns (Pharmacia Fine Chemicals). Lymphocytes eluted from the Sephadex G-10 columns were used in the lymphocyte-HAEC co-cultures and proliferation assays described below. Lymphocytes used in the syngeneic co-cultures were isolated from abdominal lymph nodes of organ donors as follows. Lymph nodes were collected under sterile conditions, injected, and flushed with sterile RPMI 1640. Lymphocytes were collected from the lymph node effluent by centrifugation, resuspended in RPMI 1640 with 10\% FCS, and counted.

HAEC-lymphocyte co-cultures and isolation of conditioned media. $5 \mathrm{~d}$ before isolating the lymphocytes (day -5 ), confluent cultures of pooled HAECs were harvested from the culture flasks with TrypsinEDTA (Gibco) and then plated at a 1:3 split ratio into either $60-\mathrm{mm}$ tissue culture dishes (Falcon 3060; Becton Dickinson Labware, Lincoln Park, NJ) pretreated with gelatin ( $1 \%$ gelatin in $\mathrm{dH}_{2}$ ), G-2500; Sigma Chemical Co.) or wells of 96-well flat-bottom plates (Falcon 3072; Becton Dickinson Labware) pretreated with human fibronectin (4 $\mu \mathrm{g} / \mathrm{cm}^{2}$; Collaborative Research Inc., Bedford, MA). The HAECs were cultured in EGM-UV supplemented with $3 \% \mathrm{FCS}$ at $37^{\circ} \mathrm{C}, 5 \%$ $\mathrm{CO}_{2}$ for $5 \mathrm{~d}$, during which time they formed confluent monolayers. On the day that the lymphocytes were prepared (day 0 ), the medium was removed from the 60 -mm dishes of the HAECs, the confluent HAECs were gently washed with RPMI 1640 , and lymphocytes prepared from individual buffy coats were added to the dishes at a ratio of 10 lymphocytes per HAEC. The lymphocyte and HAEC co-culture medium consisted of RPMI 1640 supplemented with $100 \mathrm{U} / \mathrm{ml}$ penicillin, 100 $\mu \mathrm{g} / \mathrm{ml}$ streptomycin, $25 \mathrm{mM}$ Hepes, $24 \mathrm{mM}$ sodium bicarbonate, $\mathrm{pH}$ 7.2, and $15 \%$ human AB serum (Normlcera-Plus; North American Biologicals, Inc., Miami, FL). The above medium alone (without lymphocytes) was added to the control HAECs. $24 \mathrm{~h}$ after the addition of the lymphocytes to the $60-\mathrm{mm}$ dishes of HAECs, the dishes were gently rotated and the medium containing nonadherent cells was collected. The conditioned medium was centrifuged ( $10 \mathrm{~min}, 250 \mathrm{~g}$ ) to remove the cells and then stored at $-70^{\circ} \mathrm{C}$ until it could be used for the HASMC proliferation assays (see below). All of the dishes of HAECs were washed three times with RPMI 1640 to remove any remaining lymphocytes from those dishes to which lymphocytes were added. Microscopic inspection of the washed dishes revealed the continued presence of the HAECs and few if any remaining lymphocytes (less than $0.1 \%$ of total cells). Polyadenylated RNA [poly $(\mathrm{A})^{+}$RNA] was isolated from these HAECs as described below.

Lymphocyte proliferation. On the day that the lymphocytes were isolated (day 0 ), the 96-well plates containing wells of confluent HAECs were irradiated $\left(2,000 \mathrm{rad}, \mathrm{Cs}^{137}\right)$ to prevent any further proliferation of the HAECs, the medium was removed from the wells, the wells washed with RPMI 1640, and the lymphocytes added to the wells in the co-culture medium described above. As in the co-cultures above, the lymphocytes were added to give a lymphocyte/HAEC ratio of 10:1 $\left(3 \times 10^{5}\right.$ lymphocytes/well $)$. Controls included HAECs cultured alone and lymphocytes cultured alone. Quadruplicate wells were set up for each variable. Incorporation of $\left[{ }^{3} \mathrm{H}\right]$ thymidine in co-cultures was determined after 3 and $5 \mathrm{~d}$ of co-incubation by adding $5 \mu \mathrm{Ci} / \mathrm{ml}$ of $\left[{ }^{3} \mathrm{H}\right]-$ thymidine (sp act $6.7 \mathrm{Ci} / \mathrm{mmol}$; New England Nuclear/Dupont, Boston, MA) to each well for $20 \mathrm{~h}$. The lymphocytes were then harvested onto glass fiber filter paper, using a semiautomated cell harvesting apparatus (Skatron, Inc., Sterling, VA). The filter pads were dried and relative $\left[{ }^{3} \mathrm{H}\right]$ thymidine incorporation was determined by liquid scintillation counting. Lymphocyte proliferation results were expressed in cpm or as stimulation index ( $\mathrm{SI}$ ) by dividing the activated lymphocyte incorporation by the resting lymphocyte incorporation. Both the peak and the mean lymphocyte proliferative responses between days 3 and 5 were analyzed.

HASMC proliferation to conditioned media. HASMCs were plated into flat-bottom 96 -well plates at $50 \%$ confluency $(10,000$ cells/well) in RPMI 1640 supplemented with $20 \%$ FCS (HASMC growth medium) and cultured at $37^{\circ} \mathrm{C}$ in a $5 \% \mathrm{CO}_{2}$ humidified incubator. $24 \mathrm{~h}$ later, HASMC cultures were "growth arrested" by changing the culture medium to RPMI 1640 supplemented with reduced FCS $(0.5 \%)$ for an 
additional $72 \mathrm{~h}$. The culture medium was then replaced with conditioned medium from the HAEC lymphocyte co-cultures or from the HAECs alone. The HASMCs were cultured for an additional $20 \mathrm{~h}, 5$ $\mu \mathrm{Ci} / \mathrm{ml}$ of $\left[{ }^{3} \mathrm{H}\right]$ thymidine (sp act $81 \mathrm{Ci} / \mathrm{mM}$; New England Nuclear/ Dupont, Boston, MA) was added and $4 \mathrm{~h}$ later the HASMCs were harvested on glass fiber filters using a semiautomated cell harvesting apparatus (Skatron, Inc.). The relative incorporation of $\left[{ }^{3} \mathrm{H}\right]-$ thymidine was then determined by liquid scintillation counting. All experimental points were performed in quadruplicate.

$H A E C \operatorname{Poly}(A)^{+}$RNA isolation. $20 \mathrm{~h}$ after the addition of lymphocytes to the HAECs, the lymphocytes were removed from the HAEC monolayers by multiple washings with RPMI 1640 (see above). Visual microscopic evaluation consistently demonstrated less than $0.1 \%$ of contaminating lymphocytes relative to the remaining HAECs, after washing. Poly ( $\mathrm{A}^{+}{ }^{+}$RNA was isolated from HAECs, using MicroFast Track mRNA Isolation Kits (Invitrogen, San Diego, CA ) per the manufacturer's protocol. Briefly, cell monolayers were disrupted, using a SDS-containing lysis buffer. Lysates were subjected to a proteinase $K$ digestion. The DNA was sheared and the poly $(\mathrm{A})^{+}$-containing RNA was allowed to bind to oligo(dT)-cellulose. The oligo(dT)-cellulose was then washed several times with high salt buffer followed by two low salt washes. After which, bound material was eluted with salt-free elution buffer and precipitated by the addition of $1 / 7 \mathrm{vol} 2 \mathrm{M}$ sodium acetate and $3 \mathrm{vol}$ of $100 \%$ ethanol and incubation at $-20^{\circ} \mathrm{C}$ overnight. The concentration of RNA was determined by spectrophotometry.

Reverse transcription and PCR. The amount of Poly (A) ${ }^{+}$RNA used for reverse transcription was identical for each condition ( 2 experimentals, 1 control) within an experiment and varied from $0.32-0.35 \mu \mathrm{g}$ per reaction between experiments. Poly $(\mathrm{A})^{+} \mathrm{RNA}$ was combined with oligo(dT)2-18 primer and added to a reverse transcription cocktail containing reverse transcription buffer (BRL Life Technologies, Gaithersburg, MD), dithiothreitol (BRL Life Technologies) dNTPs, RNasin (Promega Corp., Madison, WI), and M-MLV Reverse Transcriptase (BRL Life Technologies). The reaction incubated at $37^{\circ} \mathrm{C}$ for $1 \mathrm{~h}$.

Oligonucleotide primers were designed from the published human cDNA sequences of acidic and basic fibroblast growth factors (aFGF and bFGF, respectively), transforming growth factors alpha and beta (TGF- $\alpha$ and TGF- $\beta$, respectively) and both the A and B chains of PDGF (PDGF A and PDGF B, respectively) (Genbank Accession numbers M23017, J04513, K03222, X02812, X03795, and M16288, respectively) and the published human cDNA sequence of amphiregulin (AR) (24) and IL-1 $\alpha(25)$. Primers corresponding to the coding and noncoding strands were synthesized:

\begin{tabular}{|c|c|c|}
\hline & Coding & Noncoding \\
\hline $\mathrm{aFGF}$ & 5'ACCACCTTCACAGCCCTGAC3', & 5'GCTTCATACTTACTGAGCCG3'; \\
\hline bFGF & 5'TTCTTCCTGCGCATCCACCC3', & 5'ATAGCTTTCTGCCCAGGTCC3'; \\
\hline TGF $-\alpha$ & 5'CCTTGGAGAACAGCACGTCC $3^{\prime}$, & 5'GACCACTGTTTCTGAGTGGC3'; \\
\hline TGF $-\beta$ & 5'GAAGCGGAAGCGCATCGAGG3', & 5'TCCACGGCTCAACCACTGCC $3^{\prime}$; \\
\hline PDGF A & 5'GTACTGAATTTCGCCGCCAC3', & 5'GCGCACTCCAAATGCTCCTC $3^{\prime}$; \\
\hline PDGF B & 5'CTGCTGCTACCTGCGTCTGG3', & 5'GCCCACACACTCTCCTGCCG3 \\
\hline AR & 5'CCCAGAGACCGAGTTGCCCC $3^{\prime}$, & 5'TATGACTTGGCAGTGACTCC \\
\hline & AA3', $^{\prime}$ & TCATT \\
\hline
\end{tabular}

All primers were designed to cross intron-exon boundaries and were verified to be unique for the genes studied, based on the Genbank data base. Primers were tested with high cycle amplification (at least 40 cycles) and all PCR products produced single bands on agarose gel electrophoresis at the appropriate molecular weights. To confirm further the identity of the amplified cDNA, restriction enzyme analysis was performed. In general, two different endonucleases were used for each diagnosis. In all cases the appropriate fragment sizes were produced for each of the gene products studied.

$2 \mu \mathrm{l}$ of the cDNA was combined with $4 \mu \mathrm{l}$ each of $2.5-\mathrm{mM}$ coding and noncoding oligonucleotides, $1.0 \mu 110 \mathrm{mM} \mathrm{dNTPs}, 5.0 \mu \mathrm{l} 10 \times \mathrm{PCR}$ buffer ( $100 \mathrm{mM}$ Tris $\mathrm{HCl}, 500 \mathrm{mM} \mathrm{KCl}, 30 \mathrm{mM} \mathrm{MgCl}_{2}, 0.1 \%$ gelatin, pH 8.3), $33.5 \mu$ l distilled water, and 2.5 units Taq Polymerase (Perkin Elmer Cetus Corp. Norwalk, CT) to form a 50- $\mu$ PCR cocktail.

Two PCR profiles were used. 20 cycles of PCR were performed with bFGF, TGF- $\beta$, and PDGF A and B, while 30 cycles were used to amplify aFGF, TGF- $\alpha$, IL- $1 \alpha$, and AR. Each cycle consisted of $1.25 \mathrm{~min}$ $95^{\circ} \mathrm{C}$ denaturation; $1.0 \mathrm{~min} 60^{\circ} \mathrm{C}$ annealing; and $3.0 \mathrm{~min} 72^{\circ} \mathrm{C}$ polymerization steps. Initial studies failed to detect aFGF or AR mRNA in any HAEC RNA isolates. Therefore, later studies excluded analysis for these gene products.

Quantification of PCR products by HPLC. The PCR products were quantitated using a HPLC system (model 1090; Hewlett Packard Co., Palo Alto, CA) equipped with a UV detector, data handling chemstation, and a TSK DEAE-NPR analytic column (Perkin Elmer Cetus Corp., Norwalk, CT) as previously described (26) with minor modifications. 40 of the $50 \mu \mathrm{l}$ of the PCR product were utilized per injection. The mobile phase consisted of two solvents, $1 \mathrm{M} \mathrm{NaCl}$ with $25 \mathrm{mM}$ Tris- $\mathrm{HCl}$ ( solvent A) and $25 \mathrm{mM}$ Tris- $\mathrm{HCl}$ alone (solvent B), adjusted to a $\mathrm{pH}$ of 9.0 , and filtered $(0.22 \mu \mathrm{m}$ membrane $)$. Haell digested pBR322 DNA (Boehringer Mannheim Biochemicals) was used as a molecular weight marker. The separated PCR-generated cDNA products were analyzed for their absorbance peak ("HPLC area").

The ability to accurately quantitate cDNA PCR products using HPLC was confirmed at several levels in control studies using Poly (A) ${ }^{+}$RNA from HAECs. Fig. 1 is an example of an HPLC elution profile. The PCR product analyzed in this example was CDNA amplified with primers specific for TGF- $\alpha$. The abscissa is the elution time and the ordinate is the absorbance at $260 \mathrm{~nm}$ of the eluted fractions. HPLC of PCR products resulted in their elution at characteristic time points, in this case $6.34 \mathrm{~min}$.

As the goal for these experiments was to determine whether relative differences in the amounts of mRNA expressed by lymphocyte-co-cultured HAECs and control HAECs existed, no attempt was made to determine absolute numbers of mRNA copies per cell. Therefore, each set of oligo primers was tested individually to insure that a linear relationship existed between the amount of Poly $(\mathrm{A})^{+}$RNA used in the reverse transcription-PCR sequence and the amount of amplified cDNA detected by chromatography. An example of this verification is demonstrated for bFGF in Fig. 2. Varying amounts ( 2 to $40 \mu \mathrm{l}$ ) of PCR cDNA from a single PCR were quantified multiple times by HPLC (Fig. $2 A$ ). There was a linear relationship between the amount of PCR cDNA product injected and the HPLC area. When cDNA product of a single reverse transcription reaction was titrated over a broad range, each amount of cDNA input into a separate PCR reaction and quantified by HPLC, again there was a linear relationship (Fig. $2 \mathrm{~B}$ ). Finally, when Poly $(\mathrm{A})^{+}$RNA was titrated, each concentration was individually reverse-transcribed, the cDNA products individually amplified by PCR, and the PCR products quantified by HPLC, there was a linear relationship between the initial amount of Poly $(\mathrm{A})^{+} \mathrm{RNA}$ and the final amount of cDNA detected by HPLC (Fig. $2 C$ ).

To insure viability of all steps of each experimental setup, a known quantity of human $\beta$-globin mRNA (kindly donated by Dr. G. Bagby, Veterans Affairs Medical Center, Portland, OR) was added to each of the HAEC Poly (A) ${ }^{+}$RNA isolates. This mRNA was then reverse-transcribed and amplified at both cycle numbers used in this protocol with the HAEC poly (A) ${ }^{+}$RNA and quantitated by HPLC. The HPLC area of $\beta$-globin product amplified at 20 cycles and 30 cycles was $24.9 \pm 4.9$ and $84.7 \pm 21.6$, respectively. Since HAECs do not appreciably express human $\beta$-globin mRNA under any of the conditions described above, detection of the $\beta$-globin-specific PCR product for the individual Poly (A) ${ }^{+}$RNA isolates confirmed the viability of each of the steps of the mRNA isolation and detection.

Syngeneic lymphocyte-HAEC responses. To examine the response using both lymphocytes and HAECs from the same donor (syngeneic 


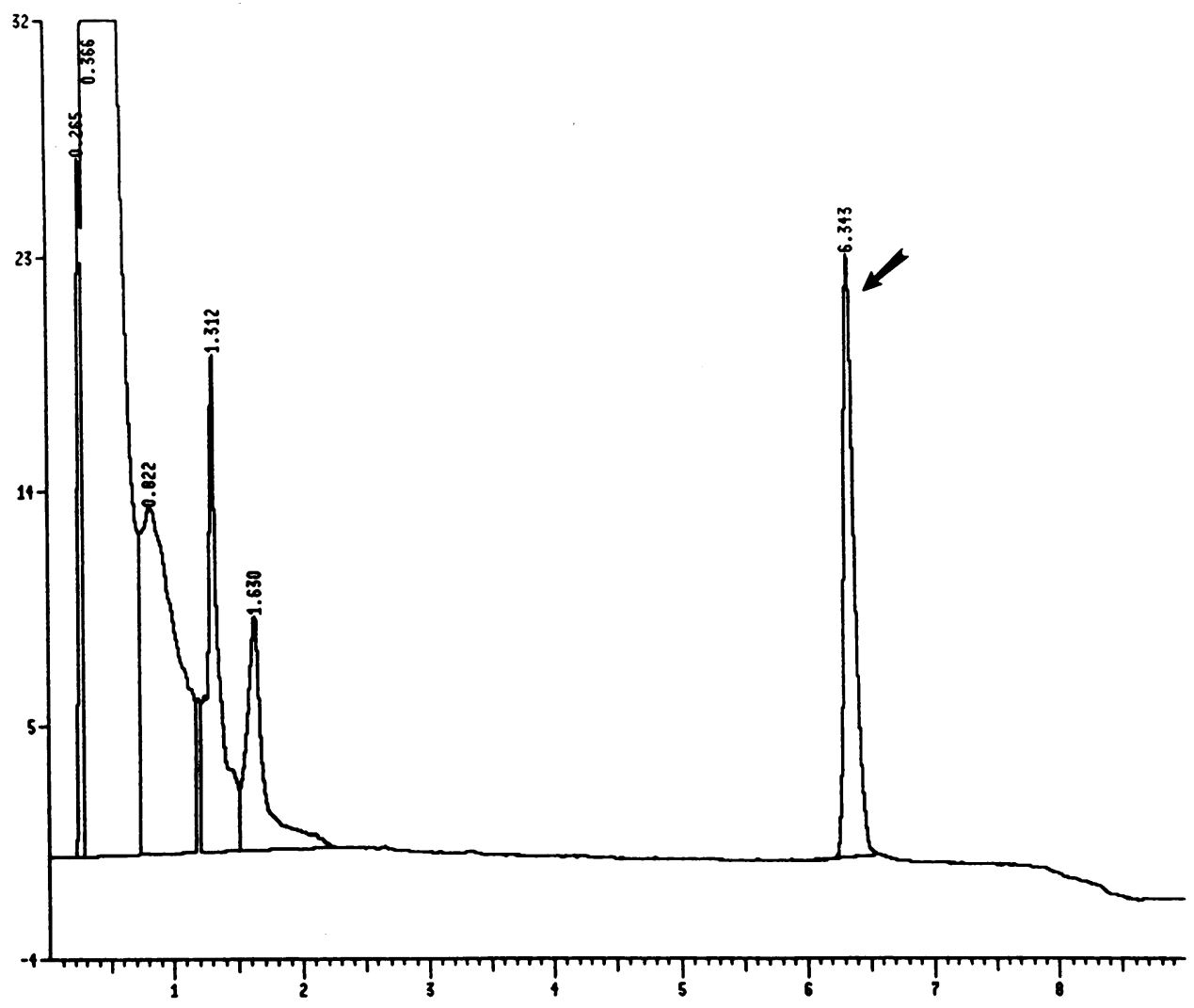

Figure 1. Typical chromatogram produced from HPLC separation of PCR products. The PCR product analyzed in this example was cDNA amplified with primers specific for TGF- $\alpha$. The abscissa is the elution time and the ordinate is the absorbance at $260 \mathrm{~nm}$ of the eluted fractions. HPLC of PCR products resulted in their elution at characteristic time points $(6.34 \mathrm{~min}$ for TGF- $\alpha$, arrow). The amount measured is proportional to the integrated area under the peak.

response), lymphocytes were isolated from abdominal lymph nodes and HAECs were isolated from thoracic aortas, as described above, from two additional organ donors. Lymphocyte proliferation to syngeneic HAECs after $5 \mathrm{~d}$ of co-culture was performed as previously described. Proliferation of the abdominal lymph node lymphocytes to a nonspecific mitogen (PHA, $12.5 \mu \mathrm{g} / \mathrm{ml}$ ) was also assessed. HAEC growth factor expression was performed $20 \mathrm{~h}$ after co-culture again, as previously described.

Data analysis. Both lymphocyte and HASMC proliferation were expressed as cpm and are a result of quadruplicate determinations that were averaged. PCR product was expressed as the HPLC area under the cDNA peak. Results from the entire group of HAEC-lymphocyte cocultures or their products (activated HAEC cultures) were compared to the entire group of HAEC cultures alone (control HAEC cultures) or their products, pairing those activated HAEC cultures with their respective control HAEC cultures. Differences were determined using Student's $t$ test for paired data, resulting in a single $P$ value for a given assay (e.g., bFGF mRNA levels). Correlations between measurements were performed using Pearson's correlation coefficient $r$. Significance was considered present at a $P$ value of $<0.05$.

\section{Results}

Allogeneic lymphocyte proliferation to HAECs. Antigen presenting cells such as endothelial cells, are known to stimulate the proliferation of lymphocytes when the lymphocytes and stimulating cells are allogeneic (27). While the MHC phenotype of the pooled HAECs used for these studies was partially known (Table I), the lymphocytes for the experiments below came from anonymous blood donors whose MHC phenotypes were unknown. To ascertain whether the lymphocytes from the separate donors were alloreactive to the HAECs, a mixed endothelial cell-lymphocyte culture was performed for each lymphocyte donor. Lymphocytes were cultured with the
HAECs and lymphocyte proliferation was assayed after 3 and 5 $\mathrm{d}$ for the incorporation of $\left[{ }^{3} \mathrm{H}\right]$ thymidine. While the magnitude varied, the lymphocytes from all 10 donors were alloreactive to the HAECs (Table II). There was no evidence of lymphocyte proliferation in response to syngeneic HAECs in the two co-cultures studied ( $\mathrm{SI}<2.0$, at $5 \mathrm{~d}$, results not shown), but both isolates responded appropriately to PHA.

$H A S M C$ proliferation in response to mixed HAEC-lymphocyte conditioned medium. If the interaction of allogeneic lymphocytes and endothelial cells stimulates endothelial cell production of smooth muscle cell growth factors, then it is possible that those growth factors would be released into the medium of the co-cultured lymphocytes and HAECs. To test this possibility, medium was collected from nine co-cultures of lymphocytes and HAECs (activated-HAEC media), added to wells of 96-well plates containing HASMCs, and assayed for its ability to stimulate HASMC proliferation. Each time activated-HAEC medium was generated, medium was also collected from HAECs cultured alone (control media). ActivatedHAEC medium from seven of the nine combinations of lymphocytes and HAECs significantly increased HASMC incorporation of $\left[{ }^{3} \mathrm{H}\right]$ thymidine, compared to that of HASMCs cultured in control medium $(P=0.014$, Fig. 3$)$. In a separate set of experiments, medium conditioned by lymphocytes alone or lymphocytes stimulated with PHA did not significantly change HASMC $\left[{ }^{3} \mathrm{H}\right]$ thymidine incorporation compared to that of HASMCs cultured in medium conditioned by HAECs alone, while, again, medium conditioned by both HAECs and lymphocytes increased $\left[{ }^{3} \mathrm{H}\right]$ thymidine incorporation (results not shown).

Allogeneic lymphocyte regulation of $H A E C$-derived growth factor $m R N A$. There are several mechanisms that could account for the increased HASMC growth stimulatory activity of 

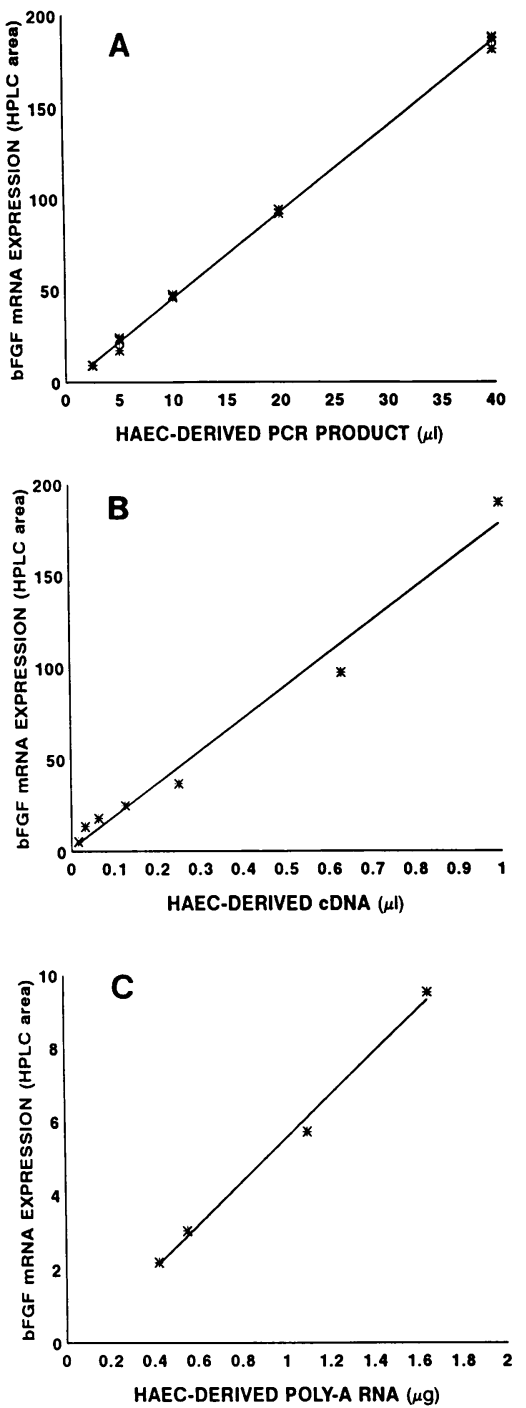

Figure 2. Example of the validation process for HPLC analysis and subsequent quantification of PCR products performed for each of the oligonucleotide primers used (in this example bFGF). ( $A$ ) Multiple volumes (concentrations) of PCR product are analyzed with several repeat measurements. (B) Several volumes of reverse transcription product are subjected to individual PCR amplification reactions and analyzed. (C) Four concentrations of Poly $(\mathrm{A})^{+}$RNA are subjected to individual reverse transcription reactions followed by individual PCR amplification reactions and analyzed. There are linear relationships at all three validation levels ( $r$ $=0.99$ ).

the media conditioned by the allogeneic lymphocytes and endothelial cells. One possible means is that the interaction of the allogeneic lymphocytes and HAECs stimulates HAEC production of mRNA for HASMC growth factors, leading to the increased synthesis and release of the growth factors. To test the hypothesis that allogeneic lymphocytes and HAECs interact so as to increase HAEC growth factor mRNA, poly $(\mathrm{A})^{+}$RNA was isolated from HAECs following co-culture with allogeneic lymphocytes (activated HAECs). The RNA was reverse-transcribed, amplified by PCR using oligonucleotide primers specific for one of a panel of growth factors, and the absorbance of the PCR product was quantitated following HPLC (see Methods). The relative quantities of PCR products generated from poly (A) ${ }^{+}$RNA isolated from control HAECs and activated HAECs was then compared for evidence of allogeneic lymphocyte regulation of the HAEC mRNA synthesis.

The poly (A) ${ }^{+}$RNA was successfully isolated from 9 of 10 HAEC cultures exposed to allogeneic lymphocytes. The matched isolates of activated and control HAEC poly (A) ${ }^{+}$ RNA were analyzed for their relative quantity of several growth factors' mRNA, including that for bFGF, TGF- $\alpha$, TGF- $\beta$, and the $A$ and $B$ chains of PDGF. The results for the individual isolates of RNA demonstrate that for all of the growth factors, in the majority of the experiments (i.e., for at least seven of the nine lymphocyte preparations), interaction of the allogeneic lymphocytes and HAECs increased the HAEC's growth factor mRNA level (Fig. 4). The relative quantities of mRNA for bFGF, TGF- $\beta$, and PDGF-A chain all increased significantly with exposure to allogeneic lymphocytes $(P<0.05)$. Although the increase in TGF- $\alpha$ and PDGF-B chain mRNA was marginally significant $(P=0.077$ and $P=0.065$, respectively), as mentioned above, in the majority of the activated HAEC cultures there was an increase compared to the control HAEC cultures. In contrast to the above mesenchymal growth factors, there was no significant increase in HAEC mRNA coding for IL- $1 \alpha$ in response to allogeneic lymphocytes. Finally, in the two syngeneic co-cultures studied there was no increase in mRNA levels coding for bFGF, TGF- $\beta$, TGF- $\alpha$, PDGF-A chain, or PDGF-B chain.

To exclude the possibility that lymphocyte poly $(\mathrm{A})^{+}$RNA was partially responsible for the increase in growth factor mRNA, the HAECs were washed extensively to remove any lymphocytes before isolating the HAEC poly $(\mathrm{A})^{+}$RNA (Methods). Secondly, poly (A) ${ }^{+}$RNA was isolated from the recovered lymphocytes, reverse-transcribed, and amplified with the same growth factor mRNA oligonucleotide primers as used for the HAEC poly (A) ${ }^{+}$RNA, and that were analyzed by agarose gel electrophoresis. In order to obtain visual bands, the PCR was performed for 40 cycles (rather than the 20 or 30 cycles for HPLC analysis), and the amount of lymphocyte poly (A) ${ }^{+}$RNA used in the reverse transcription reaction was 10fold higher than would be anticipated, based on the visual assessment of $0.1 \%$ or less remaining lymphocytes in the HAEC cultures. PCR products from reverse-transcribed control HAEC poly $(\mathrm{A})^{+}$RNA resulted in bands specific for bFGF, TGF- $\alpha$, TGF- $\beta$, and PDGF-A and PDGF-B chains (Fig. $5 A$ ) while the lymphocyte poly $(\mathrm{A})^{+}$RNA resulted in only extremely faint bands for TGF- $\beta$ and PDGF-B (Fig. $5 B$ ). It is unlikely that either of these products from lymphocyte poly (A) ${ }^{+}$RNA would be detectable at the 20-cycle PCR amplification used for the quantitative analysis. Third, two isolates of naive lymphocytes were activated with PMA; poly $(\mathrm{A})^{+}$RNA was isolated from these lymphocytes, reverse-transcribed, and amplified with the same growth factor mRNA oligonucleotide primers and amplification programs as used for the HAEC poly $(\mathrm{A})^{+} \mathrm{RNA}$; and the PCR products were quantitated using HPLC. No PCR products were detected for any of the genes studied.

In all experiments lymphocyte proliferation increased, in most experiments mRNA for the various growth factors increased (the exception was IL- $1 \alpha$ ), and in most experiments

Table I. HLA Antigens Represented in HAEC Pool*

\begin{tabular}{ccccc}
\hline Donor & HLA-A & HLA-B & HLA-C & HLA-DR \\
\hline 1 & 1,29 & 7,44 & & \\
2 & 1,2 & 35,57 & & 1,7 \\
3 & 11,32 & 14,22 & 3 & \\
4 & 2,11 & 35,57 & 4 & 4,7 \\
5 & 3,11 & 7,22 & 3 & 2 \\
6 & 2,28 & 17,51 & 1 & 5,6 \\
7 & 1,29 & 27,56 & & 5,7
\end{tabular}

* Equal numbers of HAECs isolated from the above seven donors were combined to form the HAEC pool used in these experiments. 
Table II. Proliferative Responses of Individual's PBLs to Pooled Allogeneic HAECs*

\begin{tabular}{|c|c|c|c|c|c|c|c|c|}
\hline \multirow[b]{2}{*}{$\begin{array}{l}\text { PBL } \\
\text { Donor }\end{array}$} & \multicolumn{4}{|c|}{ Day $3^{\ddagger}$} & \multicolumn{4}{|c|}{ Day 5} \\
\hline & $\begin{array}{l}\text { HAECs } \\
\text { Only }\end{array}$ & $\begin{array}{l}\text { PBLs } \\
\text { Only }\end{array}$ & $\begin{aligned} \text { PBLs } \\
+ \text { HAECs }\end{aligned}$ & $\mathrm{SI}^{\mathbf{s}}$ & $\begin{array}{l}\text { HAECs } \\
\text { Only }\end{array}$ & $\begin{array}{l}\text { PBLs } \\
\text { Only }\end{array}$ & $\begin{aligned} & \text { PBLs } \\
+ & \text { HAECs }\end{aligned}$ & SI \\
\hline 1 & $74 \pm 22^{11}$ & $357 \pm 26$ & $2,358 \pm 142$ & 6.6 & $90 \pm 7$ & $364 \pm 193$ & $3,659 \pm 622$ & 10.0 \\
\hline 2 & $172 \pm 117$ & $326 \pm 14$ & $1,975 \pm 384$ & 6.0 & $93 \pm 46$ & $204 \pm 24$ & $3,472 \pm 297$ & 17.0 \\
\hline 3 & $112 \pm 19$ & $144 \pm 21$ & $1,296 \pm 201$ & 9.0 & $76 \pm 14$ & $271 \pm 45$ & $608 \pm 101$ & 2.2 \\
\hline 4 & $179 \pm 168$ & $157 \pm 45$ & $760 \pm 50$ & 4.8 & $74 \pm 14$ & $171 \pm 30$ & $439 \pm 114$ & 2.6 \\
\hline 5 & $90 \pm 24$ & $197 \pm 33$ & $719 \pm 100$ & 3.6 & $369 \pm 43$ & $808 \pm 420$ & $1,578 \pm 106$ & 2.0 \\
\hline 6 & $116 \pm 21$ & $220 \pm 50$ & $7,794 \pm 1,960$ & 35.4 & $239 \pm 67$ & $342 \pm 122$ & $4,572 \pm 520$ & 13.4 \\
\hline 7 & $104 \pm 33$ & $264 \pm 32$ & $691 \pm 218$ & 2.6 & $89 \pm 24$ & $141 \pm 18$ & $1,029 \pm 282$ & 7.3 \\
\hline 8 & $73 \pm 42$ & $270 \pm 36$ & $489 \pm 62$ & 1.8 & $83 \pm 30$ & $177 \pm 27$ & $1,863 \pm 203$ & 10.5 \\
\hline 9 & $87 \pm 21$ & $248 \pm 40$ & $461 \pm 97$ & 1.8 & $167 \pm 29$ & $123 \pm 12$ & $829 \pm 49$ & 6.7 \\
\hline 10 & $110 \pm 50$ & $242 \pm 15$ & $413 \pm 38$ & 1.7 & $83 \pm 12$ & $168 \pm 38$ & $1,145 \pm 278$ & 6.8 \\
\hline
\end{tabular}

* Mixed HAEC-peripheral blood lymphocyte (PBL) cultures were performed as described in Methods. ${ }^{\ddagger}$ Proliferation cultures were harvested on both days 3 and 5, as described in Methods. ${ }^{8} \mathrm{SI}=$ (mean cpm PBLs + HAECs) $\div$ (mean cpm PBLs). A SI of 2.0 or greater reflects a significant difference $(P<0.05)$ in the PBL proliferative response to the allogeneic HAECs vs. PBLs alone (Student's $t$ test, unpaired data). "Results are presented as the mean cpm \pm SD. The mean cpm from "HAECs Only" was not significantly different from that of medium only.

conditioned media increased HASMC proliferation. There were no correlations between growth factor mRNA expression, HASMC proliferation, and lymphocyte proliferation.

\section{Discussion}

The data presented here demonstrate that allogeneic lymphocytes are capable of inducing HAECs to increase the expression of mRNA for a panel of growth factors known to modulate smooth muscle cell proliferation (11). These data further demonstrate that as a result of this interaction, biologically active products are produced in the culture medium that increase the proliferation of HASMC in culture. To our knowledge, this is the first demonstration that a cell-mediated allogeneic response is capable of directly regulating specific gene products in vascular target cells derived from adult human tissue.

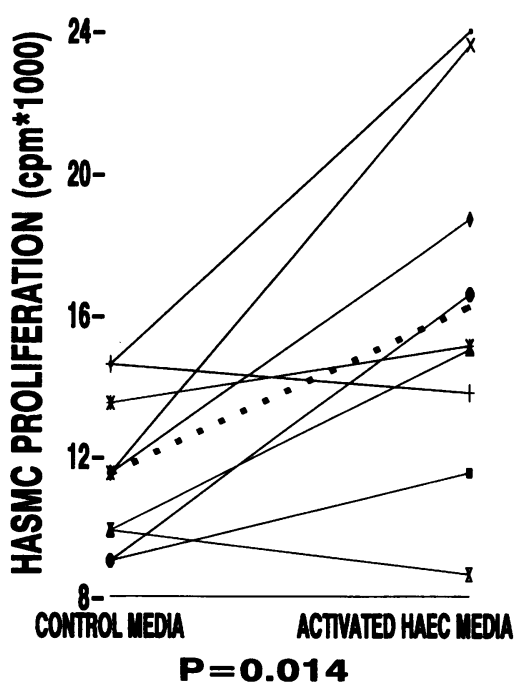

Figure 3. The HASMC proliferative response to conditioned media from the activated HAEC cultures and the control HAEC cultures. The values, presented as cpm of HASMC [ $3 \mathrm{H}]-$ thymidine incorporation, are shown for each individual experiment with the specific control connected to its respective experimental by the thin lines. The mean change for the group is shown as the heavy dotted line. As a group, there was a statistically significant increase $(P$ $=0.014)$ in HASMC

proliferation in response to media from activated HAEC cultures.
While the pathogenesis of cardiac allograft vasculopathy is unknown, for several reasons it is believed to involve an immune response of the recipient to the allogeneic donor vasculature $(1,3)$. For example, the disease process is limited to the allograft; it does not disseminate to encompass the recipient's own vessels. Yet, while an immunological process is thought to be involved, it certainly is not overtly aggressive. Histopathologic analysis reveals a low grade inflammatory process without vascular necrosis (8). We and others have proposed that allograft endothelial cells provoke an alloreactive immune response, resulting in the production of cytokines that elicit smooth muscle cell migration and proliferation $(1,3)$. In addition to their alloantigen presenting capabilities, endothelial cells are a rich source of cell growth factors $(12,15,16)$. Increased production of those cytokines may be induced by the interaction of allogeneic lymphocytes and endothelial cells, thereby stimulating the smooth muscle cell neointimal hyperplasia characteristic of CAV. Our results support this hypothesis by demonstrating that allogeneic lymphocytes and endothelial cells interact to release biologically active products that stimulate HASMC proliferation in vitro, and that allogeneic lymphocyte-HAEC co-culture increases HAEC expression of mRNA for a panel of growth factors known to modulate smooth muscle cell proliferation.

Endothelial cells are known to be capable of producing a wide variety of cytokines, including those associated with immune responses and those traditionally thought of as fibroblast or smooth muscle cell growth factors. Such cytokines include IL-1, IL-6, PDGF, TGF- $\alpha$, TGF- $\beta$, aFGF, and bFGF $(1,3$, 28 ). Besides cytokines such as IL-1 and IL-6, whose regulated synthesis and release is known to occur as part of an immune response, there is preliminary evidence of lymphocyte products regulating endothelial cell expression of PDGF $(29,30)$. For example, TNF- $\alpha$ and IL-1 can induce the expression of PDGF $(29,30)$. In contrast another activated-lymphocyte product, IFN- $\gamma$ down-regulates the production of PDGF stimulated by TNF- $\alpha$ or IL-1 (30). A question then remains as to what the overall net effect is of lymphocyte-endothelial cell interaction with regard to regulation of endothelial cell growth factor pro- 

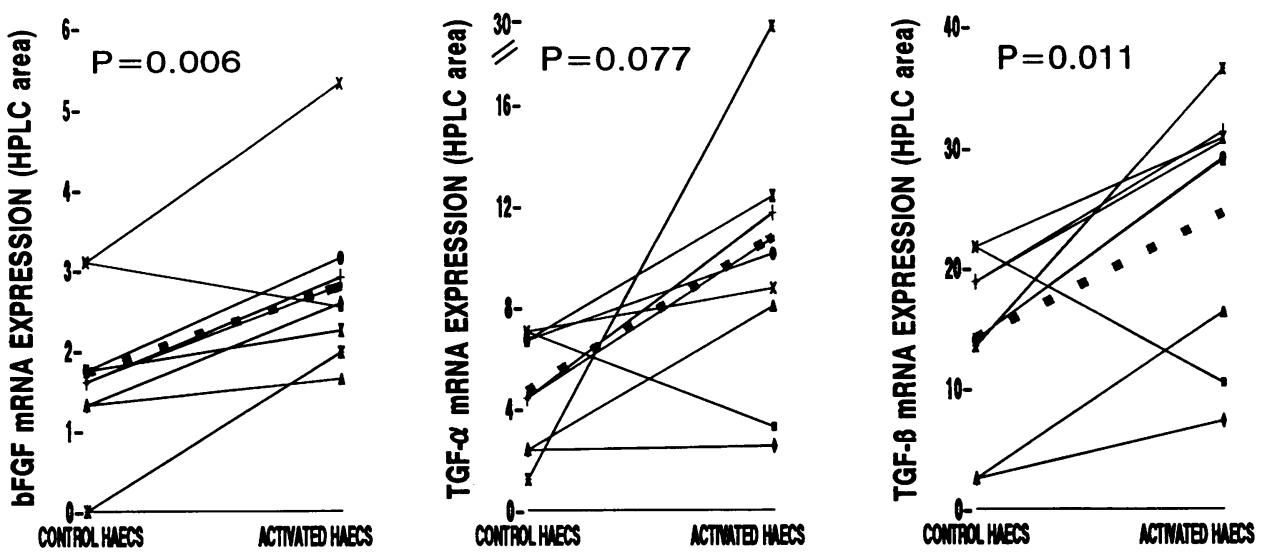

Figure 4. Quantification of growth factor mRNA from control HAECs (Control HAECs) and HAECs cocultured with allogeneic lymphocytes (Activated HAECs). The relative quantity of mRNA coding for the six genes studied, expressed as "HPLC area" (see Methods), is shown for each individual experiment with the specific control connected to its respective experimental by the thin lines. The mean change
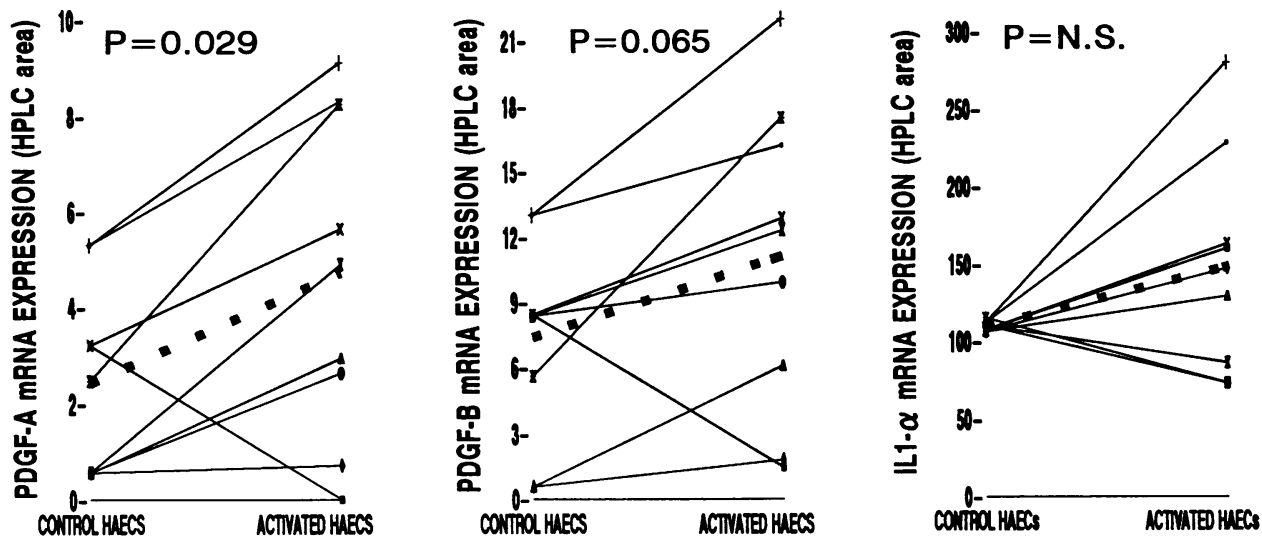

for the group is shown as the heavy dotted line. In the majority of experiments (seven out of nine for PDGF-A chain and eight out of nine for all of the others except IL- $1 \alpha$ ) co-culturing of allogeneic lymphocytes with HAECs increased the HAEC expression of growth factor mRNA. As a group, the increase in bFGF, TGF- $\beta$, and PDGF-A

mRNA levels was statistically significant, the increases in TGF- $\alpha$ and PDGF-B were only borderline significant, and there was no increase in IL-1 $\alpha$ mRNA levels.

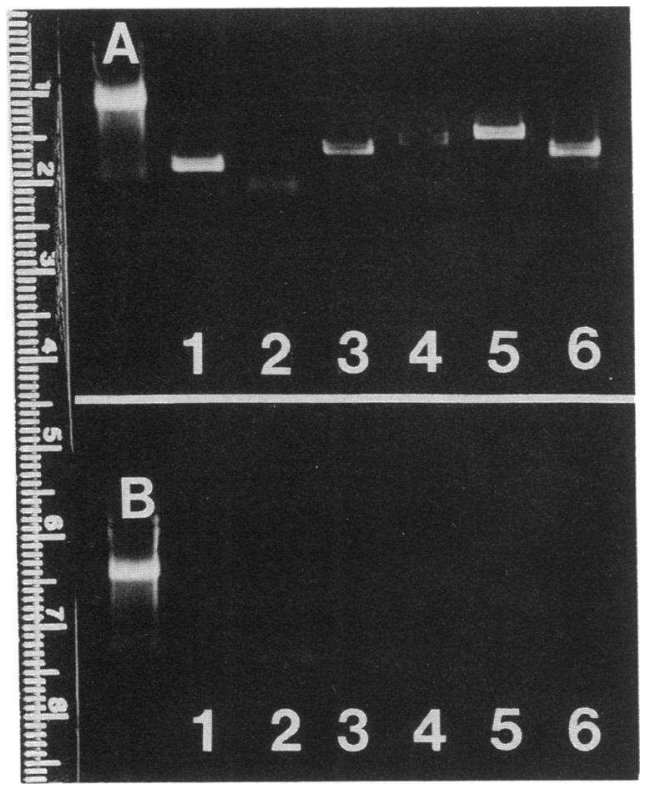

Figure 5. PCR amplification of CDNA from control HAECs $(A)$ and lymphocytes recovered from co-cultures of lymphocytes and HAECs (B). PCR amplification was performed for $\mathbf{4 0}$ cycles to give visual bands on the agarose gels shown. PCR amplification of cDNA from control HAECs resulted in products for bFGF, TGF-a, TGF-b, PDGF $A$ chain and PDGF $B$ chain, while amplification of $c D N A$ from lymphocytes resulted in only weak bands for TGF- $\beta$ and PDGF B chain. Lane 1 , bFGF; 2 , TGF- $\alpha$; 3 , TGF- $\beta$; 4 , PDGF-A; 5 , PDFG-B; $6, \beta$ globin [added to HAEC derived poly $(\mathrm{A})^{+}$RNA only]. duction. Shaddy et al. demonstrated that human umbilical artery endothelial cells, though not umbilical vein endothelial cells, expressed a "PDGF-like" protein when incubated with allogeneic lymphocytes (31). Since we had demonstrated that endothelial cells derived from adult arterial tissue (aorta) can express genes for several smooth muscle cell growth factors (32), we asked whether allogeneic lymphocytes might regulate expression of those genes in a manner that could be envisioned to stimulate the development of CAV.

Several aspects of the current study require comment. The experiments performed here utilize adult aortic endothelial cells isolated from tissue obtained at the time of cardiac donor organ retrieval. While questions regarding CAV might be ideally studied using endothelial cells isolated from coronary arteries, CAV has been seen to extend to the donor aorta, suggesting that aortic endothelial cells are not inappropriate for these studies. A pool of HAECs generated from the isolates of several donors was used, so that the results would be representative of HAEC responses in general (vs. that of a specific HAEC isolate), and so that the endothelial cells would express MHC antigens stimulatory to lymphocytes from multiple individuals. Prospective HLA matching is not performed in cardiac transplantation, so it is usual that substantial mismatches occur in the clinical setting. In fact, the lymphocytes from all 10 donors were alloreactive to the pooled HAECs as measured by their proliferation (Table II). The level of proliferation did vary, which most likely is reflective of the degree of MHC incompatibility between the lymphocyte donor and the pool of HAECs. 
Second, the ability to find a direct control for the allogeneic response in this human system is difficult. A truly direct syngeneic control would be to use PBLs from the donor, cultured with the donor's HAEC isolate. Unfortunately, due to the clinical circumstances surrounding brain death, the majority of donors are massively transfused as a result of their traumatic injury. Hence, the PBLs are usually not their own. We elected to use lymphocytes isolated from abdominal lymph nodes to maximize recovery of donor lymphocytes. Although these isolates were not co-cultured with the random endothelial cell pool, they both responded appropriately to nonspecific mitogen (PHA). Whether these lymphocytes are an appropriate control for PBLs is unclear.

Third, there was no correlation between the magnitude of the lymphocyte proliferative responses and the level of growth factor expression. The lack of significant correlation may be due to a difference in the harvest time points for the two protocols (lymphocyte proliferation was measured at 3 and $5 \mathrm{~d}$, while HAEC mRNA was isolated at $20 \mathrm{~h}$ ). Another possibility is that the intracellular signals that trigger lymphocyte proliferation do not completely overlap with those that regulate HAEC mRNA expression. For example, potentially the upregulation of HAEC mRNA expression is a result of class I HLA incompatibilities, while lymphocyte proliferation is a result of both class I and II HLA differences.

Fourth, we have demonstrated that mRNA for a variety of growth factors known to stimulate smooth muscle cell proliferation are increased as a result of the allogeneic response and that media conditioned by this response in fact stimulate smooth muscle cell proliferation. We do not know whether the specific growth factors investigated are in fact responsible singly, in combination, or not at all for the actual HASMC proliferative response demonstrated. Furthermore, it is conceivable that the responsible soluble factor(s) differ, depending on the specific allo-response (from co-culture to co-culture).

Fifth, it was assumed that the mRNA levels measured were all derived from the HAEC population, given the low numbers of contaminating cells, the lack of significant expression of these growth factor genes in lymphocytes removed from these co-cultures, and the lack of expression of these growth factor genes from PMA-activated lymphocytes. We cannot, however, be absolutely sure that the small numbers of lymphocytes specifically adhering to the HAEC pool after the extensive washing were not activated in a more specific or unique way (compared to less specific activation ) and were actively expressing a significant amount of the growth factor genes of interest. Likewise, the conditioned medium was a product of both the lymphocytes and HAECs, and it is possible that the medium contained lymphocyte-produced cytokines that effected the HASMC proliferation. This is unlikely, given the absence of a HASMC proliferative effect from medium conditioned by lymphocytes alone or lectin-stimulated lymphocytes, but, again, we cannot rule out the possibility that lymphocytes specifically activated would not produce different cytokines than lymphocytes nonspecifically activated. If our assumptions are wrong in either instance, this may be less important from a pathophysiologic and mechanistic view of CAV, where lymphocytes have been shown to be present in the vessel wall (3) and thus might be more representative of the in vivo cell-cell interactions.

Finally, while the interaction of the lymphocytes and HAECs almost consistently increased the HAECs bFGF, TGF$\alpha \& \beta$, and PDGF mRNA levels, the magnitude of the mRNA increase was modest, ranging between 50 to $150 \%$. This is in fact appropriate for how CAV is thought to progress; smooth muscle cell migration and proliferation in CAV is thought to develop chronically over a period of months to years.

The studies presented here do not exclude the potential contribution of humoral immunity to the development of CAV $(1,4)$ or the contribution of cytokines produced by the lymphocytes and macrophages that infiltrate the vessel walls in CAV (3). The results of these experiments do suggest that the interaction of circulating alloreactive recipient lymphocytes with donor endothelial cells can stimulate endothelial cell synthesis of smooth muscle cell migratory and proliferative factors that may be sufficient to lead to the development of CAV.

\section{Acknowledgments}

The authors thank the Pacific Northwest Transplant Bank for providing human tissue for cell culture, Dr. Allen M. Gown for the gift of HHF35 monoclonal antibody, Dr. Grover C. Bagby for the gift of human $\beta$-globin mRNA, Dr. John Ansel for providing assistance with initial RNA analysis, Dr. Michael Davey and Dimitri Munkirs for providing expertise in HPLC techniques, Dr. Nathan Kemalyan for his assistance in the restriction enzyme analysis, and Susan Fogg and Kimberly Wiltse for their excellent technical assistance.

This study was supported by National Institutes of Health grant HL-43369, The American Heart Association, Oregon Affiliate, and Merit Review research funds from the Department of Veterans Affairs.

\section{References}

1. Hosenpud, J. D., G. D. Shipley, and C. R. Wagner. 1992. Cardiac allograft vasculopathy: current concepts, recent developments, and future directions. $J$. Heart Lung Transplant. 11:9-23.

2. Oni, A. A., J. Ray, and J. D. Hosenpud. 1992. Coronary venous intimal thickening in explanted cardiac allografts: evidence demonstrating that transplant coronary artery disease is a manifestation of a diffuse allograft vasculopathy. Transplantation (Baltimore). 53:1247-1251.

3. Libby, P., R. N. Salomon, D. D. Payne, F. J. Schoen, and J. S. Pober. 1989. Functions of vascular wall cells related to developments of transplantation-associated coronary arteriosclerosis. Transplant. Proc. 21:3677-3684.

4. Rose, E. A., C. R. Smith, G. A. Petrossian, M. L. Barr, and K. Reemtsma. 1989. Humoral immune responses after cardiac transplantation: correlation with fatal rejection and graft atherosclerosis. Surgery (St. Louis). 106:203-207.

5. Grattan, M. T., C. E. Moreno-Cabral, V. A. Starnes, P. E. Oyer, E. B. Stinson, and N. E. Shumway. 1989. Cytomegalovirus is associated with cardiac allograft rejection and atherosclerosis. J. Am. Med. Assoc. 261:3561-3566.

6. McDonald, K., T. S. Rector, E. A. Braulin, S. H. Kubo, and M. T. Olivari. 1989. Association of coronary artery disease in cardiac transplant recipients with cytomegalovirus infection. Am. J. Cardiol. 64:359-362.

7. Laden, A. M., and R. A. Sinclair. 1971. Thickening of arterial intima in rat cardiac allografts. A light and electron microscopic study. Am. J. Pathol. 63:6984.

8. Salomon, R. N., C. C. W. Hughes, F. J. Schoen, D. D. Payne, J. S. Pober, and P. Libby. 1991. Human coronary transplantation-associated arteriosclerosis: evidence for a chronic immune reaction to activated graft endothelial cells. Am.J. Pathol. 138:791-798.

9. Goustin, A. S., E. B. Leof, G. D. Shipley, and H. L. Moses. 1986. Growth factors and cancer. Cancer Res. 46:1015-1029.

10. Shipley, G. D., W. W. Keeble, J. E. Hendrickson, R. J. Coffey Jr., and M. R. Pottelkow. 1988. Growth of normal human keratinocytes and fibroblasts in serum-free medium is stimulated by acidic and basic fibroblast growth factor. J. Cell. Physiol. 138:511-518.

11. Hoshi, H., M. Kan, J. K. Chen, and W. L. Mckeehan. 1988. Comparative endocrinology-paracrinology-autocrinology of human adult large vessel endothelial and smooth muscle cells. In Vitro Cell. Dev. Biol. 24:309-314.

12. Ross, R. 1986. The pathogenesis of atherosclerosis-an update. N. Engl. J. Med. 314:488-500.

13. Barrett, T. B., and E. P. Benditt. 1989. Platelet derived growth factor gene expression in human atherosclerotic plaque. Proc. Natl. Acad. Sci. USA. 85:2810-2814. 
14. Ross, R., J. Masuda, E. W. Raines, A. M. Gown, S. Katsuda, M. Sasahara, L. T. Malden, H. Masuko, and H. Sato. 1990. Localization of PDGF-B protein in macrophages in all phases of atherogenesis. Science (Wash. DC). 248:10091012.

15. Sato, Y., and D. B. Rifkin. 1988. Autocrine activities of basic fibroblast growth factor: regulation of endothelial cell movement, plasminogen activator synthesis, and DNA synthesis. J. Cell. Biol. 107:1199-1205.

16. Schweigerer, L., G. Neufeld, J. Friedman, J. A. Abraham, J. C. Fiddes, and D. Gospodarowicz. 1987. Capillary endothelial cells express basic fibroblast growth factor, a mitogen that promotes their own growth. Nature (Lond.). 325:257-259.

17. Hosenpud, J. D., C. Sunwen, and C. R. Wagner. 1991. Cytomegalovirusinduced regulation of major histocompatibility complex class I antigen expression in human aortic smooth muscle cells. Transplantation (Baltimore). 52:896903.

18. Wagner, C. R., R. M. Vetto, and D. R. Burger. 1985. Subcultured human endothelial cells can function independently as fully competent antigen-presenting cells. Hum. Immunol. 13:33-47.

19. Jaffe, E. A., L. W. Hoyer, and R. L. Nachman. 1973. Synthesis of antihemophilic factor antigen by cultured human endothelial cells. J. Clin. Invest. 52:2754-2756.

20. Voyta, J. C., D. P. Via, C. E. Butterfield, and B. R. Zetter. 1984. Identification and isolation of endothelial cells based on their increased uptake of acetylated low density lipoprotein. J. Cell. Biol. 99:2034-2040.

21. Tsukada, T., D. Tippens, D. Gordon, R. Ross, and A. M. Gowen. 1987. HHF35, a muscle-actin-specific monoclonal antibody: I. Immunocytochemical and biochemical characterization. Am. J. Pathol. 126:51-60.

22. Skalli, O., P. Ropraz, and G. Benzonana. 1986. A monoclonal antibody against alpha-smooth muscle actin. A new probe for smooth muscle differentiation. J. Cell. Biol. 103:2787-2796.

23. Thieme, T. R., S. H. Hefeneider, C. R. Wagner, and D. R. Burger. 1987. Recombinant murine and human IL- $\alpha$ bind to human endothelial cells with an equal affinity, but have an unequal ability to induce endothelial cell adherence of lymphocytes. J. Immunol. 139:1173-1178.

24. Plowman, G. D., J. M. Green, V. L. McDonald, M. G. Neubauer, C. M. Disteche, G. J. Todaro, and M. Shoyab. 1990. The amphiregulin gene encodes a novel epidermal growth factor-related protein with tumor-inhibitory activity. Mol. Cell. Biol. 10:1969-1981.

25. Furutani, Y., M. Natake, T. Fukui, M. Ohue, H. Nomura, M. Yamada, and S. Nakamura. 1986. Complete nuceotide sequence of the gene for human interleukin 1 alpha. Nucleic Acids Res. 14:3167-3172.

26. Katz, E. D., and M. W. Dong. 1990. Rapid analysis and purification of polymerase chain reaction products by high-performance liquid chromatography. Biotechniques. 8:546-554.

27. Hirschberg, H., S. A. Evensen, T. Henriksen, and E. Thorsby. 1975. The human mixed lymphocyte-endothelium culture interaction. Transplantation (Baltimore). 19:495-504.

28. Ikeda, U., M. Ikeda, T. Oohara, A. Oguchi, T. Kamitani, Y. Tsuruya, and S. Kano. 1991. Interleukin 6 stimulates growth of vascular smooth muscle cells in a PDGF-dependent manner. Am. J. Physiol. 260:H1713-H1717.

29. Hajjar, K. A., D. P. Hajjar, R. L. Silverstein, and R. L. Nachman. 1987. Tumor necrosis factor-mediated release of platelet-derived growth factor from cultured endothelial cells. J. Exp. Med. 166:235-245.

30. Suzuki, H., K. Shibano, M. Okane, I. Kono, Y. Matsui, K. Yamane, and H. Kashiwagi. 1989. Interferon- $\gamma$ modulates messenger RNA levels of c-sis (PDGF-B chain ), PDGF-A chain, and IL- $1 \beta$ genes in human vascular endothelial cells. Am. J. Pathol. 134:35-43.

31. Shaddy, R. E., J. C. Hansen, and C. G. Cowley. 1992. Effects of T-cells on platelet-derived growth factor-like protein secretion from endothelial cells. $J$. Heart Lung Transplant. 11:48-57.

32. Morris, T. E., P. A. Mattox, G. D. Shipley, C. R. Wagner, and J. D. Hosenpud. 1993. Pattern of cytokine messenger RNA expression in human aortic endothelial cells is different from that of human umbilical vein endothelial cells. Transplant. Immunol. In press. 